DR. DARRICK KONG LI (Orcid ID : 0000-0002-1983-0165)

DR. FATEH BAZERBACHI (Orcid ID : 0000-0003-4599-2042)

Article type : Original Articles

Editor : Michelle Long

\title{
Normal Liver Stiffness and Influencing Factors in Healthy Children: An Individual Participant Data Meta-analysis
}

Darrick K. Li ${ }^{1}$, Muhammad Rehan Khan ${ }^{2}$, Zhen Wang ${ }^{3}$, Voranush Chongsrisawat ${ }^{4}$, Panida Swangsak $^{4}$, Ulrike Teufel-Schäfer ${ }^{5}$, Guido Engelmann ${ }^{6}$, Imeke Goldschmidt ${ }^{7}$, Ulrich Baumann $^{7,8}$, Daisuke Tokuhara ${ }^{9}$, Yuki Cho' ${ }^{9}$, Marion Rowland ${ }^{10}$, Anders B. Mjelle ${ }^{11,12,13}$, Grant A. Ramm ${ }^{14}$, Peter J. Lewindon ${ }^{15}$, Peter Witters ${ }^{16}$, David Cassiman ${ }^{17}$, Ioana M. Ciuca ${ }^{18}$, Larry D. Prokop ${ }^{3}$, Samir Haffar ${ }^{19}$, Kathleen E. Corey ${ }^{1}$, M. Hassan Murad ${ }^{3}$, Katryn N. Furuya $^{20}$, Fateh Bazerbachi ${ }^{1}$

'Division of Gastroenterology, Department of Medicine, Massachusetts General Hospital, Boston, MA USA

2Division of Pediatric Gastroenterology, Hepatology, and Nutrition, University of Illinois College of Medicine at Peoria, Children's Hospital of Illinois, Peoria, IL USA

${ }^{3}$ Evidence-Based Practice Center, Robert D. and Patricia E. Kern Center for the Science of Health Care Delivery, Mayo Clinic, Rochester, MN USA

4Department of Pediatrics, Faculty of Medicine, Chulalongkorn University and King Chulalongkorn Memorial Hospital, Bangkok, Thailand

This article has been accepted for publication and undergone full peer review but has not been through the copyediting, typesetting, pagination and proofreading process, which may lead to differences between this version and the Version of Record. Please cite this article as doi:

$\underline{10.1111 / \mathrm{LIV} .14658}$

This article is protected by copyright. All rights reserved 
5Department of Pediatrics and Adolescent Medicine, Medical Center University of Freiburg, Faculty of Medicine, University of Freiburg, Freiburg, Germany

${ }^{6}$ Department of Pediatrics, Lukas Hospital, D-41464 Neuss, Germany

${ }^{7}$ Division of Paediatric Gastroenterology and Hepatology, Hannover Medical School, Hannover, Germany

8Institute of Immunology and Immunotherapy, University of Birmigham, United Kingdom

${ }^{9}$ Department of Pediatrics, Osaka City University Graduate School of Medicine, Osaka, Japan

${ }^{10}$ School of Medicine and Medical Science, University College Dublin, Dublin, Ireland

${ }^{11}$ Department of Pediatric and Adolescent Medicine, Haukeland University Hospital, Bergen, Norway

${ }^{12}$ Department of Clinical Medicine, University of Bergen, Bergen, Norway

${ }^{13}$ National Centre for Ultrasound in Gastroenterology, Department of Medicine, Haukeland University Hospital, Bergen, Norway

${ }^{14}$ Hepatic Fibrosis Group, QIMR Berghofer Medical Research Institute, Herston, QLD 4006, Australia

${ }^{15}$ Department of Gastroenterology, Hepatology and Liver Transplant, Queensland Children's Hospital, Brisbane, Australia

${ }^{16}$ Department of Paediatrics, University Hospitals Leuven, Leuven, Belgium

${ }^{17}$ Department of Gastroenterology-Hepatology and Metabolic Center, University of Leuven, Leuven, Belgium

${ }^{18}$ Pediatrics Department, University of Medicine and Pharmacy "Victor Babes", Timisoara, Romania

19Digestive Center for Diagnosis and Treatment, Damascus, Syrian Arab Republic.

${ }^{20}$ Department of Pediatrics, University of Wisconsin - Madison School of Medicine and Public Health, Madison, WI USA

Word Count: 4998 
Number of Figures and Tables: 7

\section{List of Abbreviations:}

ALT alanine aminotransferase

AST aspartate aminotransferase

BMI body mass index

CAP controlled attenuation parameter

GGT gamma-glutamyl transferase

IPD individual participant data

$\mathrm{kPa} \quad$ kilopascal

LSM liver stiffness measurement

NAFLD non-alcoholic fatty liver disease

TE transient elastography

WFL weight for length

\section{Corresponding Author:}

Fateh Bazerbachi, MD

Assistant in Medicine, Instructor in Medicine

Harvard Medical School

Division of Gastroenterology

Department of Medicine

Massachusetts General Hospital

55 Fruit Street

Wang $539 \mathrm{~K}$

Boston, MA 02114

Tel: +1 6177260607

Email: fbazerbachi@mgh.harvard.edu 
Conflict of Interest Statement: The authors of this paper certify that they have NO affiliations with or involvement in any organization or entity with any financial or non-financial interest in the subject matter or materials discussed in this manuscript.

Financial or Grant Support: None.

This article is protected by copyright. All rights reserved 


\section{Abstract}

Background \& Aims: Although transient elastography is used to determine liver stiffness as a surrogate to hepatic fibrosis, the normal range in children is not well defined. We performed a systematic review and individual participant data meta-analysis to determine the range of liver stiffness in healthy children and evaluate the influence of important biological parameters.

Methods: We pooled data from 10 studies that examined healthy children using transient elastography. We divided 1702 children into two groups: $\geq 3$ years (older group) and $<3$ years of age (younger group). Univariate and multivariate linear regression models predicting liver stiffness were conducted.

Results: After excluding children with obesity, diabetes, or abnormal liver tests, 652 children were analyzed. Among older children, mean liver stiffness was $4.45 \mathrm{kPa}(95 \%$ confidence interval 4.34-4.56), and increased liver stiffness was associated with age, sedation status, and $S$ probe use. In the younger group, the mean liver stiffness was $4.79 \mathrm{kPa}(95 \%$ confidence interval 4.46-5.12), and increased liver stiffness was associated with sedation status and Caucasian race. In a subgroup analysis, hepatic steatosis on ultrasound was significantly associated with increased liver stiffness. We define a reference range for normal liver stiffness in healthy children as $2.45-5.56 \mathrm{kPa}$.

Conclusions: We have established transient elastography-derived liver stiffness ranges for healthy children and propose an upper limit of liver stiffness in healthy children to be 5.56 $\mathrm{kPa}$. We have identified increasing age, use of sedation, probe size and presence of steatosis on ultrasound as factors that can significantly increase liver stiffness.

Keywords: transient elastography, FibroScan, liver stiffness, healthy controls, steatosis

Lay Summary: Measurement of liver stiffness is increasingly used as a non-invasive method to assess the degree of liver fibrosis. While normal ranges of liver stiffness for healthy adults have been established, the normal values for healthy children are not well 
defined. Our study establishes normal ranges for healthy children and also identifies factors that may increase liver stiffness in this population.

This article is protected by copyright. All rights reserved 


\section{Introduction}

Although liver biopsy remains the gold standard for the assessment of hepatic fibrosis, its invasiveness, sampling bias, and the need for sedation/anesthesia may limit its use, particularly in children. The presence of hepatic fibrosis holds prognostic significance as its severity is strongly associated with the development of cirrhosis and consequent morbidity and mortality. Pediatric liver disease is increasing in prevalence among children and adolescents, driven by the growing epidemic of childhood obesity and non-alcoholic fatty liver disease (NAFLD) ${ }^{1}$. As such, non-invasive methods to evaluate hepatic fibrosis in children have become increasingly utilized and important in clinical practice.

Since 2003, transient elastography (TE) has been used as a non-invasive tool for liver stiffness measurement (LSM), a surrogate marker for hepatic fibrosis. TE is a rapid and noninvasive technique that is based upon the principle that the propagation velocity of a wave through a homogenous tissue is proportional to its elasticity, which itself is correlated with the degree of hepatic fibrosis. The operator uses an ultrasound transducer that measures the speed at which vibration of mild amplitude and low frequency transmits through the liver. Due to the ease of application, TE has become an established tool in the diagnosis and prospective monitoring of hepatic fibrosis in a variety of chronic liver diseases in adults ${ }^{2}$. With the widening use of this non-invasive technique, there has been increased interest in determining the normal LSM range in healthy individuals. While the normal LSM range has been identified in adults $^{3}$, no systematic review has evaluated the normal values for LSM in children and there is only data available from a small number of studies in healthy children ${ }^{4-}$ 10.

In this systematic review and individual participant data (IPD) meta-analysis, we aimed to define the normal range of TE-derived LSM, as well as identify factors influencing this range by synthesizing studies reporting the measurements in ostensibly healthy children.

This article is protected by copyright. All rights reserved 


\section{Methods}

We developed a protocol a priori and followed the guidance of Preferred Reporting Items for Systematic Reviews and Meta-Analysis of Individual Participant Data statement ${ }^{11}$. For details, a checklist is provided in Supplemental Information.

\section{Data Sources and Search Strategies}

An experienced librarian searched the following databases with guidance from the study's principal investigators: EBM Reviews - Cochrane Central Register of Controlled Trials April 2020, EBM Reviews - Cochrane Database of Systematic Reviews 2005 to May 29, 2020, Embase 1974 to 2020 May 29, Ovid MEDLINE(R) and Epub Ahead of Print, In-Process \& Other Non-Indexed Citations and Daily 1946 to May 29, 2020. The search included human studies of liver elastography as measured by TE in children and infants published in English, French, or Spanish. Details of the search strategy are available in Supplemental Information.

After the removal of duplicates and studies that evaluated LSM in non-healthy children, we identified a total of 66 entries (papers and abstracts). Contact information was collected if available in the entry or internet-based search engines.

\section{Inclusion Criteria}

Studies were included according to the following criteria. (1) studies of transient elastography as measured by FibroScan ${ }^{\circledR}$ (Echosens, Paris, France) that explicitly described the recruitment of apparently healthy individuals, as well as studies that used comparative control arms of healthy recruits; (2) individuals were less than 18 years of age; (3) the LSM examination was performed with either a standard small $(\mathrm{S} 1, \mathrm{~S} 2)$ or standard medium (M) probe. The application of the probe was at the discretion of the original study center; and (4) technically good examinations were performed according to the manufacturer's instructions.

\section{Exclusion Criteria}


Studies were excluded if they (1) included patients with known liver disease (hepatic steatosis discovered incidentally on abdominal ultrasound was not considered an exclusion criterion); (2) did not record the type of probe that was used for LSM measurements; (3) used newer technologies for TE including FibroTouch ${ }^{\circledR}$ (Hisky Medical Technologies Co. Ltd, Wuxi, China).

\section{Data Extraction}

Authors of screened studies potentially fulfilling the inclusion criteria were provided with the inclusion and exclusion criteria and invited to offer related individual participant data. The list of investigators who provided data sets is provided in Supplemental Information.

\section{Study Group Analysis}

The cohort was divided into two groups: $\geq 3$ years of age (older group) and $<3$ years of age (younger group) at the time of testing. This age was chosen as a cut off as a consensus discussion among the study authors, as it was felt that certain anthropomorphic measurements did not have validity above or below this age. As such, BMI was calculated for children older than 3 years of age, and weight for length (WFL) was calculated for children younger than 3 years of age. Z-scores were calculated using the website https://www.quesgen.com/BMIPedsCalc.php. The outcome of interest was LSM evaluated by FibroScan $®$ expressed in kilopascals $(\mathrm{kPa})$. For our primary analysis, we excluded individuals that were obese, carried a diagnosis of diabetes mellitus, or who had incidentally abnormal liver function tests (LFTs). We defined obesity in children in accordance with the Center for Disease Control guidance, as being greater than the $95^{\text {th }}$ percentile for BMI for age (BMI z-score or WFL z-score > 1.64) ${ }^{12}$. Abnormal LFTs were defined as ALT > 33 IU/L for males and $A L T>25 \mathrm{IU} / \mathrm{L}$ for females, according to the American College of Gastroenterology guidelines, and cutoffs for AST levels were chosen similar to that of $A L T^{13}$. A rigorous approach was followed so that if the the individual participant data did not specifically designate the status of diabetes in the participant, or if liver enzymes were not reported, the participant was censored from the primary analysis). 


\section{Assessment of Methodologic Quality}

Two reviewers (DKL, FB) assessed the methodologic quality of all included studies by applying a modified tool of the Newcastle-Ottawa Scale adapted to our cohort study ${ }^{14}$. We kept items focused on the representativeness of the cases and ascertainment of outcome and removed items that related to the selection of the controls and comparability. This tool has been applied in many previous publications ${ }^{3,15-18}$, with good consistency among reviewers. The assessment of methodologic quality for the 10 included cohorts and the overall risk of bias for these cohorts is found in Supplemental Information.

\section{Statistical Analysis and Regression Model Analysis}

For descriptive analysis, we reported means, standard deviation, and $95 \%$ confidence intervals $(95 \% \mathrm{Cl})$ for continuous variables and percentages for dichotomized variables unless otherwise specified.

To determine the reference range for LSM in healthy children of all ages, we first removed outliers using a method based on robust nonlinear regression with a $1 \%$ false discovery rate $^{19}$. After outliers were removed, we defined the upper limit of normal to be the $90^{\text {th }}$ percentile of LSM readings and the lower limit of normal as the $2.5^{\text {th }}$ percentile.

To evaluate the association between LSM and confounding factors, we constructed 2-level, mixed effect, linear, random-intercept, regression models with unconstructed covariance between the random effects. Observations without providing all the adjusted factors were deemed as missing and were ignored in the analysis. All statistical analyses were conducted using STATA version 14.1 (StataCorp LP, College Station, TX). 


\section{Results}

\section{Description of Included Studies}

The flow diagram for this individual participant data meta-analysis is shown in Figure 1. Our search identified 3010 entries. Reviewers (FB, SH, DKL) examined all entries. We identified 66 studies as potentially appropriate to be included if enough data were made available. Emails were sent to corresponding and primary authors of unique entries (abstracts and articles) to obtain individual participant data tables. Fifty-five authors were not able to provide data, and one study was excluded because of the use of Fibrotouch ${ }^{\circledR}$.

The study included a total of 10 cohorts, with a total of 1702 participants $4-8,10,20-23$. The breakdown of these studies and references, as well as their overall risk of bias, is outlined in Supplemental Information.

\section{Baseline Characteristics}

Of the 1702 total individuals, 201 individuals were excluded from the primary analysis due to having obesity (BMI or WFL z-score > 1.64), diabetes mellitus, or abnormal LFTs ${ }^{24}$. An additional 849 individuals were excluded because of missing information regarding diabetes status or LFTs. In our primary analysis cohort, 588 (90.2\%) were in the older group and 64 were $(9.8 \%)$ were in the younger group. The baseline characteristics of each group are shown in Table 1.

A description of the probes used for LSM determination is provided in Supplemental Table 1. Overall, the M probe was used for LSM in the majority of older children ( $n=268 ; 50.66 \%$ ), while the $\mathrm{S} 1$ probe was used for the majority of younger children $(n=59 ; 92.19 \%)$.

\section{Liver Stiffness Measurement Ranges in Apparently Healthy Children}

The mean LSM for older children was $4.45 \mathrm{kPa} \pm 1.31 \mathrm{kPa}(95 \% \mathrm{Cl} 4.34-4.56)$ and is shown in Figure 2A and Table 2, stratified by sex. Among the older group, mean LSM was significantly different between the sexes $(p=0.015)$. In this group, males and females had 
similar age $(p=0.41)$. The mean LSM for the younger group was $4.79 \mathrm{kPa} \pm 1.34 \mathrm{kPa}(95 \%$ $\mathrm{Cl}$ 4.46-5.12) and was significantly different between sexes $(p=0.043)$ as shown in Figure 2B. The average LSM for all children combining both categories was $4.48 \mathrm{kPa} \pm 1.32 \mathrm{kPa}$ (95\% Cl 4.38-4.58). Histograms for LSM values for older children and younger children are provided in Supplemental Figures 1 and 2.

Within this cohort, there were 9 children (all in the older group) who had evidence of steatosis on ultrasound. These individuals with "lean NAFLD" had a mean LSM of $6.78 \mathrm{kPa}$ (95\% Cl 2.88-10.68).

In the older group, mean LSM for Caucasian children was significantly greater than that for Asian children (4.66 vs. $4.08 \mathrm{kPa}, \mathrm{p}<0.0001)$. This was also statistically significant in the younger group of children (4.92 vs. $3.33 \mathrm{kPa}, \mathrm{p}=0.041)$.

We also performed a subgroup analysis for pre-adolescent (3-11 years old) and adolescent ( $\geq 11$ years old) children. Mean LSM for pre-adolescent and adolescent children were 4.42 $\mathrm{kPa} \pm 1.32 \mathrm{kPa}(95 \% \mathrm{Cl} 4.27-4.56)$ and $4.49 \mathrm{kPa} \pm 1.30 \mathrm{kPa}(95 \% \mathrm{Cl} 4.33-4.65)$, respectively. Histograms of LSM values for pre-adolescent and adolescent children are provided in Supplemental Figures $\mathbf{3}$ and $\mathbf{4}$. Additional stratification by sex demonstrated a trend toward a difference in LSM between male and female pre-adolescent children $(p=0.065)$ and adolescent children $(p=0.097)$ (Supplemental Table 2).

\section{Reference Range of LSM in Healthy Children}

We next derived a reference range for LSM based on this cohort and determined it to be 2.45-5.56 kPa (see Methods for details).

\section{Regression Analysis Model of Normal Liver Stiffness Measurement}

Next, we sought to identify the impact of baseline characteristics on LSM. Univariate analysis demonstrated that for older children, age, male sex, and sedation status were 
significantly associated with increased LSM (Supplemental Table 3). These variables were not significantly associated with changes in LSM in children $<3$ years old.

Next, a multivariate regression model, adjusting for confounding factors influencing LSM was developed for both age groups. The regression coefficient represents the kPa change in liver stiffness. The results of this analysis are summarized in Table 3. For older children, age, sedation status during $T E$, and $S$ probe use were associated with a statistically significant increase in LSM. The other adjusted factors (male sex, Caucasian race, BMI z-score) had no significant effect on LSM. For younger children, only Caucasian race and sedation during TE were associated with a statistically significant increase in LSM. As relayed in methods, this regression analysis included LSM values of individuals who had available data for all the adjusted factors, and those individuals who were missing any of these values were censored from the analysis.

Another regression model, including only those children with information regarding the presence or absence of steatosis on ultrasonography ( $n=216$; Table 4), was constructed. All of the children with such available data were $\geq 3$ years old. In this analysis, we found that the presence of hepatic steatosis on ultrasonography was significantly associated with increased LSM [coefficient 2.578 (standard error 0.507); $p<0.0001$ ].

Figure 3 summarizes the major results from the primary analysis of our IPD meta-analysis.

\section{Sensitivity Analysis Including All Individuals in IPD Cohort}

To explore the robustness of our results when expanded to include all individuals that were in our IPD cohort, we performed an additional sensitivity analysis where we removed the stringent exclusion criteria applied to the IPD models described previously. The resulting cohort included 1627 children $\geq 3$ years old and 75 children $<3$ years old. The baseline characteristics of this cohort are shown in Supplemental Table 4. Notably, $15.8 \%$ of older children and $12.0 \%$ of younger children in this cohort were obese. Moreover, $13.6 \%$ of older 
children had evidence of hepatic steatosis on ultrasound, and $6.6 \%$ of the older children carried a diagnosis of diabetes mellitus. The mean LSMs for older and younger children, stratified by sex, is shown in Supplemental Table 5. Among older children, we also found that the mean LSM increased gradually from healthy children to children with hepatic steatosis on ultrasound, and children with both hepatic steatosis on ultrasound and diabetes mellitus (Supplemental Table 6).

The results of a multivariate regression model, adjusting for confounding factors that influence LSM in both age groups, are summarized in Supplemental Table 7. As in our primary analysis, age and sedation status were significantly associated with an increase in LSM in the older group. However, male sex and BMI z-score were associated with increased LSM in this sensitivity analysis while Caucasian race was not. The mean LSM for obese patients (BMI Z-score > 1.64) was $4.71 \pm 1.41 \mathrm{kPa}(95 \% \mathrm{Cl} 4.53-4.89)$. A histogram of LSM values for obese patients is shown in Supplemental Figure 5.

Results of the sensitivity analysis for younger children were identical to our primary analysis. In addition, a multivariate regression model including only those children with information regarding the presence or absence of steatosis on ultrasonography demonstrated that hepatic steatosis was significantly associated with increased LSM (Supplemental Table 8).

A subset of individuals included in this larger cohort also had information regarding their diabetes mellitus status, all older children $(n=459)$. Of these, 25 children carried a diagnosis of diabetes. Although it was surmised in all cohorts, that children included as healthy were de facto non-diabetics, to provide an additional layer of rigorous sensitivity analysis, we evaluated those who carried a diagnosis of diabetes, and a trend toward increased LSM in the presence of diabetes mellitus was noted, not reaching statistical significance $(p=0.075$; Supplementary Table 9).

This article is protected by copyright. All rights reserved 
Overall, the findings of our sensitivity analysis demonstrate that the findings in our primary analysis, particularly the identification of increasing age and sedation status as factors that increase LSM in healthy children, remain robust in this larger, more inclusive cohort.

This article is protected by copyright. All rights reserved 


\section{Discussion}

Liver stiffness measurement (LSM) with transient elastography (TE) has become a standard non-invasive tool in the risk stratification of liver disease. While LSM is a surrogate of liver fibrosis, multiple confounding factors have been shown to influence the LSM in adults, including waist circumference, presence of diabetes mellitus, increased AST level, dyslipidemia, and systolic blood pressure in adults ${ }^{3}$. In children, TE studies mostly focused on identifying cutoffs for advanced fibrosis or cirrhosis in those with underlying chronic liver disease ${ }^{25,26}$. However, few studies have addressed the concept of normal liver stiffness in children or the effects of anthropomorphic parameters or comorbid conditions on LSM in reportedly healthy children (i.e., children not afflicted with liver disease). As such, there is no consensus with regards to the normal range of LSM in children. To address this unmet need, after a systematic review, we implemented an individual participant data meta-analysis accounting for these factors with sufficient power to determine a reference range for LSM as well as the effects of potential confounding factors on LSM in healthy children.

In our multivariate regression analysis, we first demonstrated that increasing age is associated with higher LSM among children $\geq 3$ years old. There have been mixed results from prior studies in regard to the effect of age on $\mathrm{LSM}^{5,9}$, which may reflect differences in examination conditions, the study sample size, or the approach to probe choice. Increasing LSM in children with respect to increasing age has recently been identified using other forms of liver stiffness measurement, including MR elastography ${ }^{27}$. The mechanisms for this are unclear at this point but may be related to metabolic, vascular, and microstructural changes occurring during maturation ${ }^{28,29}$, which have been observed in animal models ${ }^{30}$. In this large study, after controlling for other important variables, we find that age is significantly associated with increases in TE-derived LSM.

An important finding from our analysis that was consistent in both age groups studied was the significant association between sedation and increased LSM. Increased LSM due to sedation was first described in Goldschmidt et al. ${ }^{4}$, and the finding remains robust after 
controlling for confounding variables in our multivariate regression analysis. In our IPD, those individuals who underwent sedation primarily received propofol. It is thought that the increase in LSM may be related to propofol-related increased splanchnic blood flow ${ }^{31}$. The effects of other forms of sedation, including benzodiazepines, nitrous oxide, or opioids on LSM remain open questions at this time. However, our findings suggest that results of TE in a sedated child is not an accurate reflection of intrinsic LSM.

We also noted a significant association between the probe used and LSM. Specifically, we noted that the use of an S probe (S1, S2) may lead to an overestimation of LSM compared to use of an $\mathrm{M}$ probe, which has been suggested in previous studies ${ }^{32}$.

We also demonstrated that the presence of hepatic steatosis on ultrasound is associated with a significant increase in LSM. The impact of hepatic steatosis on LSM has been a controversial topic with conflicting results in the adult literature, depending on the degree of fibrosis and the etiology of any underlying chronic liver disease. In presumably healthy individuals, several studies have demonstrated a significant increase in LSM in those with hepatic steatosis on ultrasound compared to those without steatosis $3,33-35$. In our study, we have extended this observation to the pediatric population.

While mean LSM in males was significantly higher than females in both older and younger age groups, male sex was not significantly associated with an increase in LSM based on our multivariate regression model. In contrast, the multivariate regression in our sensitivity analysis that included obese children, those with diabetes, and those with abnormal LFTs, did demonstrate that male sex was significantly associated with increased LSM. Several small studies in the pediatric population have noted an increased LSM in male children, though in most of these studies, the difference was particularly notable in adolescents ${ }^{8,9,36}$. While a biological mechanism for this difference is not entirely clear, previous conjecture has suggested that estrogens and other ovarian hormones may inhibit the production of extracellular matrix by hepatic stellate cells ${ }^{37}$. Whether or not this same dynamic is valid in 
vivo remains unknown. In our primary analysis, we show a non-statistically significant trend toward increased LSM in males in both pre-adolescent and adolescent children. Ultimately, though we cannot comment definitively on the contribution of the male sex to LSM based on our results, a similar observation is seen in the adult population ${ }^{3}$. Moreover, we could not control for all potential confounding variables, which include surreptitious alcohol use and medications, particularly amongst adolescent children, as these were not captured in the pooled datasets.

In light of a rising incidence and prevalence of pediatric liver disease driven by NAFLD, these results become important. In a seminal study, Schwimmer et al. examined liver biopsies of children between the ages of 2 and 19 who underwent an autopsy identified NAFLD in $13 \%$ of subjects with prevalence increasing with age ${ }^{38}$. A recent systematic review and meta-analysis pooling studies of reported prevalence of NAFLD based on any diagnostic method including non-invasive means reported a $7.6 \%$ prevalence of NAFLD in the general pediatric population and $34 \%$ in pediatric obesity clinics ${ }^{1}$. Among individuals in the entire IPD cohort that had information regarding the presence or absence of steatosis on ultrasound, $59(16.8 \%)$ had evidence of hepatic steatosis, consistent with what has been reported previously. As the use of TE increases for monitoring liver disease in the pediatric population, our results show that the presence of steatosis may increase LSM and may need to be factored in the interpretation of results.

In addition to NAFLD, the prevalence of diabetes mellitus is also increasing among the pediatric population, driven in large part by the rise in childhood obesity. By intentional design, our primary analysis excluded children with diabetes. However, we capitalized on a large dataset that provided data in children with diabetes, by performing sensitivity analysis which identified a trend toward increased LSM in the presence of diabetes mellitus. The same observation has been demonstrated in the adult population ${ }^{3}$. Importantly, this trend toward increased LSM in patients with diabetes may suggest early underlying, and undetected fibrosis, as the metabolic syndrome has been associated with advanced liver 
fibrosis among pediatric NAFLD patients ${ }^{39}$. Our finding warrants further evaluation in larger cohorts.

The impact of hepatic inflammation (as determined by an elevated ALT) on LSM is well described in the adult population ${ }^{40}$ and has recently become appreciated in the pediatric population, particularly in patients with minimal or no fibrosis at baseline ${ }^{41}$. In our study, we were unable to include biochemical indices of hepatic inflammation, including $A L T$, in our regression model due to a limited amount of individual participant data reporting these values. However, we excluded all patients with elevated ALT from our primary analysis. Thus, the likelihood of occult hepatic inflammation in our cohort is low and incorporating ALT into our model would likely not have yielded a significant result. Nevertheless, cautious interpretation of LSM in the setting of an elevated ALT remains warranted.

The strength of our study derives from its large cohort of individuals from multiple centers that were selected after establishing an a priori protocol. Moreover, stringent inclusion and exclusion criteria were applied to exclude potential occult chronic liver disease, which may have confounded our results. Given the size of our cohort, we were also able to propose a reference range for LSM in healthy children based on rigorous statistical principles. As such, a LSM of greater than $5.56 \mathrm{kPa}$ should alert the clinician to the presence of occult liver disease and prompt further investigation. In addition, our regression analysis conclusions are robust because the data were analyzed for a given LSM only if all variables of the regression analysis were available, and as such, each variable was therefore controlled. Another important strength of our study is that we calculated the z-score for height, weight, and BMI for every individual in our analysis, even if it had not been calculated in the original data set. The z-score is widely regarded as the best system for the analysis and presentation of anthropometric data in the pediatric population ${ }^{42}$. However, the use of the $z-$ score has been inconsistent in other studies of LSM in the pediatric population and may have resulted in bias and erroneous associations. The use of z-scores dramatically 
simplifies not only clinical interpretation but also statistical analysis given that it is a normalized variable that factors in age.

Our study has multiple inherent limitations. First, though the investigators identified each individual as healthy, we cannot fully rule out the presence of occult hepatic fibrosis or other undiagnosed liver disease ${ }^{24}$. Though we assumed no clinically significant fibrosis in the individuals in our cohort, given the retrospective nature of the data used and that none of the participants underwent a liver biopsy, this assumption may not ultimately be accurate. However, given that liver biopsy was not offered to these participants due to ethical considerations, this remains a clinically reasonable assumption. Second, our data did not capture certain information including biochemical indices of inflammation as well as medications, both of which may be contributing to the overall picture. However, this is potentially a lesser concern in a reported cohort of healthy children. Third, the determination of steatosis was performed with ultrasound for which there are numerous limitations, including decreased sensitivity, particularly compared to the use of controlled attenuation parameter $(C A P)^{43}$. However, the prevalence of hepatic steatosis detected on ultrasound in our cohort is reflective of what is known from previous studies. Fourth, ethnicities, including black and Hispanic populations, were not represented in this data set despite the large cohort size, and so the applicability of our findings to these groups remains to be determined. Finally, we did not evaluate the reproducibility of TE in this study. While few studies have examined the reproducibility of TE in children ${ }^{4,10}$, it has been shown to be less than what is clinically acceptable. In one study, over $25 \%$ of children exhibited a difference of $>1 \mathrm{kPa}$ between repeat measurements ${ }^{10}$. Thus, while our study establishes the normal LSM ranges as determined by TE in children, further studies are needed to specifically optimize the reproducibility of TE in this patient population.

In conclusion, we establish normal LSM ranges for children based on a large and diverse study population of healthy children. Our multivariate regression analysis demonstrates that age, sedation, hepatic steatosis, and S probe use influence LSM in children and should be 


\section{Acknowledgements}

The authors would like to recognize the Ratchadaphiseksomphot Fund (RA 58/034), Faculty of Medicine, Chulalongkorn University and the Thailand Research Fund. The authors also recognize funding for data supplied from Rowland et al. (HRA-2014-PHS-662).

This article is protected by copyright. All rights reserved 


\section{References}

1. Anderson EL, Howe LD, Jones HE, Higgins JPT, Lawlor DA, Fraser A. The prevalence of non-alcoholic fatty liver disease in children and adolescents: A systematic review and meta-analysis. PLoS One. 2015;10(10). doi:10.1371/journal.pone.0140908

2. Ferraioli $G$, Filice $C$, Castera $L$, et al. WFUMB guidelines and recommendations for clinical use of ultrasound elastography: Part 3: Liver. Ultrasound Med Biol. 2015;41(5):1161-1179. doi:10.1016/j.ultrasmedbio.2015.03.007

3. Bazerbachi F, Haffar S, Wang Z, et al. Range of Normal Liver Stiffness and Factors Associated With Increased Stiffness Measurements in Apparently Healthy Individuals. Clin Gastroenterol Hepatol. 2019;17(1):54-64.e1. doi:10.1016/j.cgh.2018.08.069

4. Goldschmidt I, Streckenbach C, Dingemann C, et al. Application and limitations of transient liver elastography in children. J Pediatr Gastroenterol Nutr. 2013;57(1):109113. doi:10.1097/MPG.0b013e31829206a0

5. Tokuhara D, Cho Y, Shintaku H. Transient Elastography-Based Liver Stiffness AgeDependently Increases in Children. PLoS One. 2016;11(11):e0166683. doi:10.1371/journal.pone.0166683

6. Lewindon PJ, Balouch F, Pereira TN, et al. Transient liver elastography in unsedated control children: Impact of age and intercurrent illness. J Paediatr Child Health. 2016;52(6):637-642. doi:10.1111/jpc.13151

7. Cho Y, Tokuhara D, Morikawa H, et al. Transient Elastography-Based Liver Profiles in a Hospital-Based Pediatric Population in Japan. PLoS One. 2015;10(9):e0137239. doi:10.1371/journal.pone.0137239

8. Mjelle AB, Mulabecirovic A, Havre RF, et al. Normal liver stiffness values in children: A comparison of three different elastography methods. J Pediatr Gastroenterol Nutr. 2019;68(5):706-712. doi:10.1097/MPG.0000000000002320

9. Engelmann G, Gebhardt C, Wenning D, et al. Feasibility study and control values of transient elastography in healthy children. Eur J Pediatr. 2012;171(2):353-360. doi:10.1007/s00431-011-1558-7

10. Rowland M, McGee A, Broderick A, et al. Repeatability of transient elastography in 
children. Pediatr Res. May 2020. doi:10.1038/s41390-020-0916-4

11. Stewart LA, Clarke M, Rovers M, et al. Preferred reporting items for a systematic review and meta-analysis of individual participant data: The PRISMA-IPD statement. JAMA. 2015;313(16):1657-1665. doi:10.1001/jama.2015.3656

12. Centers for Disease Control and Prevention. Defining Childhood Obesity.

13. Kwo PY, Cohen SM, Lim JK. ACG Clinical Guideline: Evaluation of Abnormal Liver Chemistries. Am J Gastroenterol. 2017;112(1):18-35. doi:10.1038/ajg.2016.517

14. Murad MH, Sultan S, Haffar S, Bazerbachi F. Methodological quality and synthesis of case series and case reports. BMJ evidence-based Med. 2018;23(2):60-63. doi:10.1136/bmjebm-2017-110853

15. Bazerbachi F, Haffar S, Sugihara T, et al. Peribiliary cysts: A systematic review and proposal of a classification framework. BMJ Open Gastroenterol. 2018;5(1):e000204. doi:10.1136/bmjgast-2018-000204

16. Haffar S, Bazerbachi F, Prokop L, Watt KD, Murad MH, Chari ST. Frequency and prognosis of acute pancreatitis associated with fulminant or non-fulminant acute hepatitis A: A systematic review. Pancreatology. 2017;17(2):166-175. doi:10.1016/j.pan.2017.02.008

17. Bazerbachi F, Haffar S, Szarka LA, et al. Secretory diarrhea and hypokalemia associated with colonic pseudo-obstruction: A case study and systematic analysis of the literature. Neurogastroenterol Motil. 2017;29(11). doi:10.1111/nmo.13120

18. Jawoosh M, Haffar S, Deepak P, et al. Volvulus of the ileal pouch-anal anastomosis: a meta-narrative systematic review of frequency, diagnosis, and treatment outcomes. Gastroenterol Rep. 2019;7(6):403-410.

19. Motulsky HJ, Brown RE. Detecting outliers when fitting data with nonlinear regression - A new method based on robust nonlinear regression and the false discovery rate. BMC Bioinformatics. 2006;7(1):123. doi:10.1186/1471-2105-7-123

20. Chongsrisawat $\mathrm{V}$, Swangsak $\mathrm{P}$, Treeprasertsuk $\mathrm{S}$. Controlled attenuation parameter values in healthy Thai children. J Pediatr Gastroenterol Nutr. 2016;63(S2):S60.

21. Witters $P$, De Boeck K, Dupont $L$, et al. Non-invasive liver elastography (Fibroscan) for 
detection of cystic fibrosis-associated liver disease. J Cyst Fibros. 2009;8(6):392-399. doi:10.1016/j.jcf.2009.08.001

22. Menten R, Leonard A, Clapuyt P, Vincke P, Nicolae AC, Lebecque P. Transient elastography in patients with cystic fibrosis. Pediatr Radiol. 2010;40(7):1231-1235. doi:10.1007/s00247-009-1531-z

23. Ciuca I, Pop L, Popa Z, et al. Liver elasticity in CF associated liver disease. J Cyst Fibros. 2012;11:S112.

24. Takyar V, Nath A, Beri A, Gharib AM, Rotman Y. How healthy are the "Healthy volunteers"? Penetrance of NAFLD in the biomedical research volunteer pool. Hepatology. 2017;66(3):825-833. doi:10.1002/hep.29247

25. Behairy BE-S, Sira MM, Zalata KR, Salama E-SE, Abd-Allah MA. Transient elastography compared to liver biopsy and morphometry for predicting fibrosis in pediatric chronic liver disease: Does etiology matter? World J Gastroenterol. 2016;22(16):4238-4249. doi:10.3748/wjg.v22.i16.4238

26. Lee CK, Perez-Atayde AR, Mitchell PD, Raza R, Afdhal NH, Jonas MM. Serum biomarkers and transient elastography as predictors of advanced liver fibrosis in a United States cohort: the Boston children's hospital experience. J Pediatr. 2013;163(4):1058-64.e2. doi:10.1016/j.jpeds.2013.04.044

27. Etchell E, Jugé L, Hatt A, Sinkus R, Bilston LE. Liver Stiffness Values Are Lower in Pediatric Subjects than in Adults and Increase with Age: A Multifrequency MR Elastography Study. Radiology. 2017;283(1):222-230. doi:10.1148/radiol.2016160252

28. Johnson TN, Tucker GT, Tanner MS, Rostami-Hodjegan A. Changes in liver volume from birth to adulthood: A meta-analysis. Liver Transplant. 2005;11(12):1481-1493. doi:10.1002/tt.20519

29. Pauleau G, Sandoz B, Thollon L, Serre T, Brunet C. Anthropometric characterization of the child liver. Surg Radiol Anat. 2010;32(8):767-775. doi:10.1007/s00276-0100675-8

30. Yarpuzlu B, Ayyildiz M, Tok OE, Aktas RG, Basdogan C. Correlation between the mechanical and histological properties of liver tissue. J Mech Behav Biomed Mater. 
2014;29:403-416. doi:10.1016/j.jmbbm.2013.09.016

31. Meierhenrich R, Gauss A, Mühling B, et al. The effect of propofol and desflurane anaesthesia on human hepatic blood flow: a pilot study. Anaesthesia.

2010;65(11):1085-1093. doi:10.1111/j.1365-2044.2010.06504.x

32. Pradhan F, Ladak F, Tracey J, Crotty P, Myers RP. Feasibility and reliability of the FibroScan S2 (pediatric) probe compared with the M probe for liver stiffness measurement in small adults with chronic liver disease. Ann Hepatol. 2013;12(1):100107. doi:10.1016/s1665-2681(19)31391-2

33. Fung J, Lee CK, Chan M, et al. Defining normal liver stiffness range in a normal healthy Chinese population without liver disease. PLoS One. 2013;8(12):e85067. doi:10.1371/journal.pone.0085067

34. Colombo S, Belloli L, Zaccanelli M, et al. Normal liver stiffness and its determinants in healthy blood donors. Dig Liver Dis. 2011;43(3):231-236.

doi:10.1016/j.dld.2010.07.008

35. Cheng PN, Chiu YC, Chiu HC, Chien SC. The application of liver stiffness measurement in residents without overt liver diseases through a community-based screening program. Med (United States). 2016;95(12):e3193.

doi:10.1097/MD.0000000000003193

36. Jain V, Poddar U, Negi TS, et al. Utility and accuracy of transient elastography in determining liver fibrosis: a case-control study. Eur J Pediatr. January 2020. doi:10.1007/s00431-019-03561-y

37. Yasuda M, Shimizu I, Shiba M, Ito S. Suppressive effects of estradiol on dimethylnitrosamine-induced fibrosis of the liver in rats. Hepatology. 1999;29(3):719727. doi:10.1002/hep.510290307

38. Schwimmer JB, Deutsch R, Kahen T, Lavine JE, Stanley C, Behling C. Prevalence of fatty liver in children and adolescents. Pediatrics. 2006;118(4):1388-1393. doi:10.1542/peds.2006-1212

39. Ting YW, Wong SW, Anuar Zaini A, Mohamed R, Jalaludin MY. Metabolic Syndrome Is Associated With Advanced Liver Fibrosis Among Pediatric Patients With Non- 
alcoholic Fatty Liver Disease. Front Pediatr. 2019;7:491. doi:10.3389/fped.2019.00491

40. Arena U, Vizzutti F, Corti G, et al. Acute viral hepatitis increases liver stiffness values measured by transient elastography. Hepatology. 2008;47(2):380-384. doi:10.1002/hep.22007

41. Raizner A, Shillingford N, Mitchell PD, et al. Hepatic inflammation may influence liver stiffness measurements by transient elastography in children and young adults. $J$ Pediatr Gastroenterol Nutr. 2017;64(4):512-517. doi:10.1097/MPG.0000000000001376

42. Colan SD. The why and how of Z scores. J Am Soc Echocardiogr. 2013;26(1):38-40. doi:10.1016/j.echo.2012.11.005

43. Karlas $T$, Petroff $D$, Sasso $M$, et al. Individual patient data meta-analysis of controlled attenuation parameter (CAP) technology for assessing steatosis. J Hepatol. 2017;66(5):1022-1030. doi:10.1016/j.jhep.2016.12.022 


\begin{tabular}{|c|c|c|c|c|c|c|c|c|}
\hline 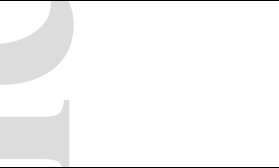 & $\begin{array}{l}\text { Patients } \\
\text { with } \\
\text { available } \\
\text { data }\end{array}$ & $\begin{array}{c}\% \text { (\# of } \\
\text { observations) }\end{array}$ & Median & IQR & $\begin{array}{l}\text { Patients } \\
\text { with } \\
\text { available } \\
\text { data }\end{array}$ & $\begin{array}{c}\% \text { (\# of } \\
\text { observations) }\end{array}$ & Median & IQR \\
\hline Male & 588 & $53.9 \%(317)$ & & & 64 & $57.8 \%(37)$ & & \\
\hline Caucasian & 482 & $58.9 \%(284)$ & & & 62 & $95.2 \%(59)$ & & \\
\hline Sedated & 525 & $9.7 \%(51)$ & & & 64 & $43.8 \%(28)$ & & \\
\hline $\begin{array}{l}\text { Steatosis on } \\
\text { ultrasound }\end{array}$ & 216 & $4.2 \%(9)$ & & & 4 & $0.0 \%(0)$ & & \\
\hline Age, yr & 588 & & 10.05 & $5.97-13.26$ & 64 & & 2.16 & $1.51-2.94$ \\
\hline Height z-score & 588 & & 0.08 & $-0.81-0.91$ & & & & \\
\hline Weight z-score & 588 & & 0.02 & $-0.76-0.63$ & & & & \\
\hline BMI z-score & 588 & & -0.04 & $-0.81-0.59$ & & & & \\
\hline WFL z-score & & & & & 64 & & -0.1 & $-0.86-0.62$ \\
\hline Hemoglobin, g/dL & 210 & & 13.1 & $12.5-13.9$ & 4 & & 11.8 & $10.1-12.5$ \\
\hline ALT, IU/L & 252 & & 14.0 & $11.3-20.0$ & 19 & & 18.0 & $16.0-22.0$ \\
\hline AST, IU/L & 213 & & 22.0 & $18.0-27.0$ & 3 & & 34.0 & $32.0-38.0$ \\
\hline GGT, IU/L & 211 & & 13.0 & $10.0-17.0$ & 18 & & 9.0 & $8.0-11.3$ \\
\hline Albumin, $\mathrm{g} / \mathrm{dL}$ & 192 & & 4.4 & 4.0-4.6 & 3 & & 4.1 & $3.7-4.1$ \\
\hline $\begin{array}{l}\text { Total bilirubin, } \\
\mathrm{mg} / \mathrm{dL}\end{array}$ & 224 & & 0.41 & $0.30-0.60$ & 19 & & 0.23 & $0.18-0.35$ \\
\hline
\end{tabular}

Table 1. Baseline characteristics of children $\geq 3$ years and $<3$ years old in the primary analysis cohort.

This article is protected by copyright. All rights reserved 


\begin{tabular}{|l|c|c|c|c|c|}
\hline \multicolumn{1}{|c|}{ Group } & $\begin{array}{c}\text { Individuals, } \\
\mathrm{n}\end{array}$ & Mean, $\mathrm{kPa}$ & $\mathrm{SD}$ & $95 \% \mathrm{Cl}$ & $p$ value \\
\hline $\mathbf{2}$ 3 years old & & & & & \\
\hline Male & 317 & 4.57 & 1.47 & $4.41-4.73$ & 0.015 \\
\hline Female & 271 & 4.31 & 1.08 & $4.18-4.44$ & \\
\hline Total & 588 & 4.45 & 1.31 & $4.34-4.56$ & \\
\hline$<\mathbf{3}$ years old & & & & & \\
\hline Male & 37 & 5.08 & 1.37 & $4.62-5.54$ & 0.043 \\
\hline Female & 27 & 4.4 & 1.2 & $3.92-4.87$ & \\
\hline Total & 64 & 4.79 & 1.34 & $4.46-5.12$ & \\
\hline
\end{tabular}

Table 2. Results of LSMs in children $\geq 3$ years of age and $<3$ years old.

This article is protected by copyright. All rights reserved 


\begin{tabular}{|l|c|c|c|c|}
\hline \multicolumn{1}{|c|}{ Factor } & Coefficient & SE & $95 \% \mathrm{Cl}$ & $p$ value \\
\hline Significant factors increasing LSM $(\geq \mathbf{3}$ years old) & & \\
\hline Age, per year & 0.05 & 0.016 & $0.018-0.082$ & 0.002 \\
\hline Sedation status & 0.588 & 0.200 & $0.195-0.981$ & 0.003 \\
\hline S probe & 0.655 & 0.223 & $0.218-1.092$ & 0.003 \\
\hline Nonsignificant factors ( $\geq \mathbf{3}$ years old) & & & \\
\hline Caucasian race & 0.105 & 0.209 & $-0.304-0.514$ & 0.615 \\
\hline Male sex & 0.169 & 0.116 & $-0.059-0.397$ & 0.146 \\
\hline BMI z-score, per point & -0.021 & 0.643 & $-0.112-0.069$ & 0.651 \\
\hline \multicolumn{2}{|c|}{} & & & \\
\hline Significant factors increasing LSM $(<3$ year old) & & & \\
\hline Caucasian race & 1.419 & 0.654 & $0.138-2.700$ & 0.03 \\
\hline Sedation status & 1.111 & 0.281 & $0.561-1.661$ & 0 \\
\hline Nonsignificant factors (<3 years old) & & & \\
\hline Male sex & 0.366 & 0.286 & $-0.196-0.927$ & 0.202 \\
\hline Age, per year & -0.215 & 0.198 & $-0.604-0.173$ & 0.277 \\
\hline Weight for length z-score, per point & -0.069 & 0.086 & $-0.237-0.010$ & 0.424 \\
\hline
\end{tabular}

*Adjustment for probe used not performed with $<3$ year old group as nearly all had LSM performed with S probe.

Table 3. Factors influencing the results of LSMs in children $\geq 3$ years of age and $<3$ years of age. 


\begin{tabular}{|l|c|c|c|c}
\hline \multicolumn{1}{c|}{ Factor } & Coefficient & SE & $95 \% \mathrm{Cl}$ & $p$ value \\
\hline \multicolumn{2}{|c|}{ Significant factors increasing LSM } & & & \\
\hline Steatosis on ultrasound & 2.578 & 0.507 & $1.585-3.571$ & $<0.001$ \\
\hline Nonsignificant factors & & & & \\
\hline Male sex & 0.291 & 0.191 & $-0.084-0.666$ & 0.128 \\
\hline Caucasian race & -0.006 & 0.391 & $-0.772-0.760$ & 0.987 \\
\hline Age, per year & 0.05 & 0.026 & $-0.001-0.102$ & 0.056 \\
\hline BMl z-score, per point & -0.054 & 0.078 & $-0.206-0.099$ & 0.489 \\
\hline Sedation status & -0.878 & 0.805 & $-2.456-0.699$ & 0.275 \\
\hline
\end{tabular}

*Adjustment for probe used not performed in this regression analysis as all patients had LSM with M probe

Table 4. Multivariate regression analysis of factors influencing the results of LSMs in children $\geq 3$ years of age with information regarding the presence or absence of hepatic steatosis on ultrasound.

This article is protected by copyright. All rights reserved 
Identification

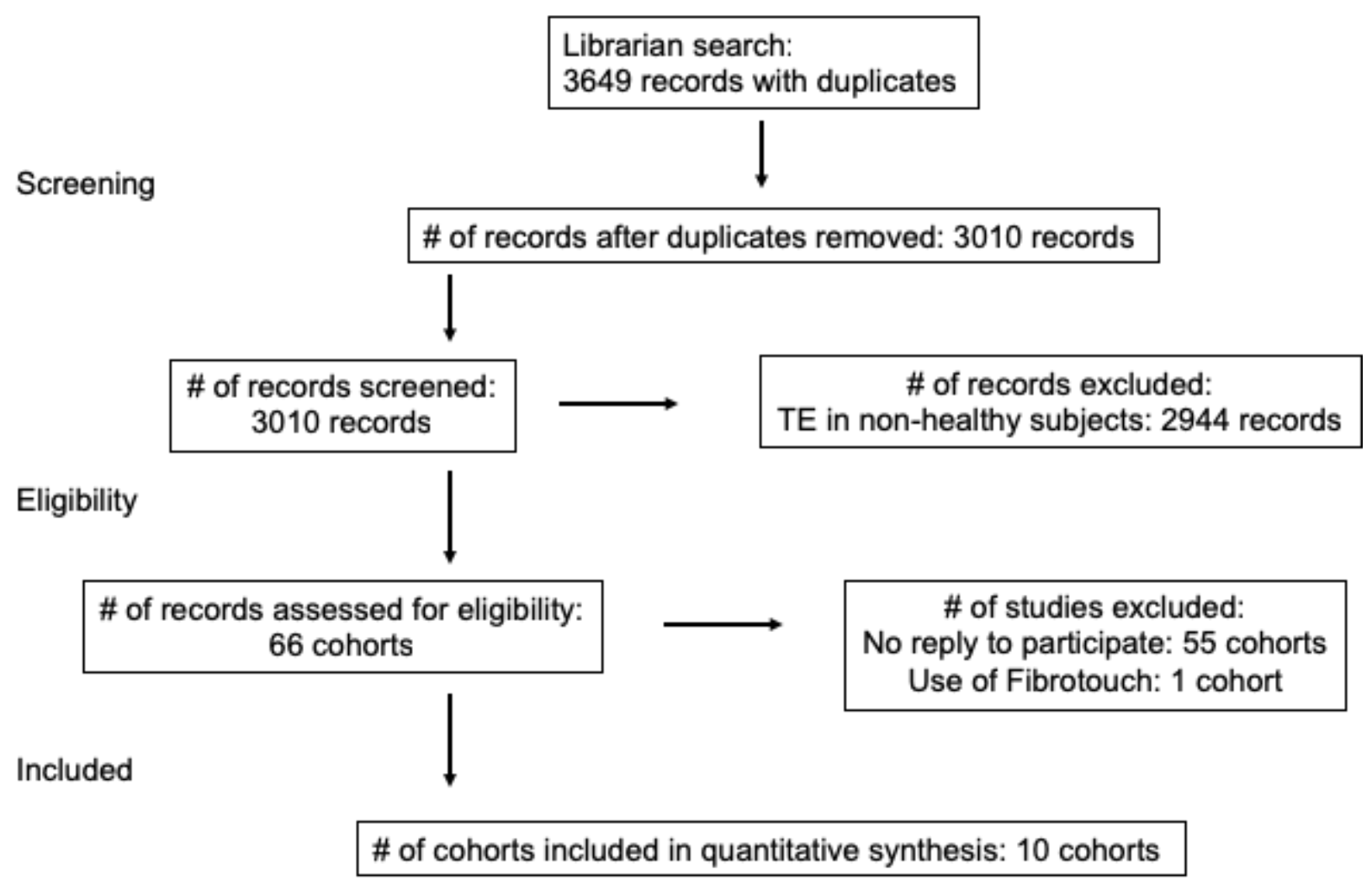

Figure 1. Flow diagram through the different phases of the individual participant data meta-analysis. 
A

LSM ( $\geq 3$ years old)

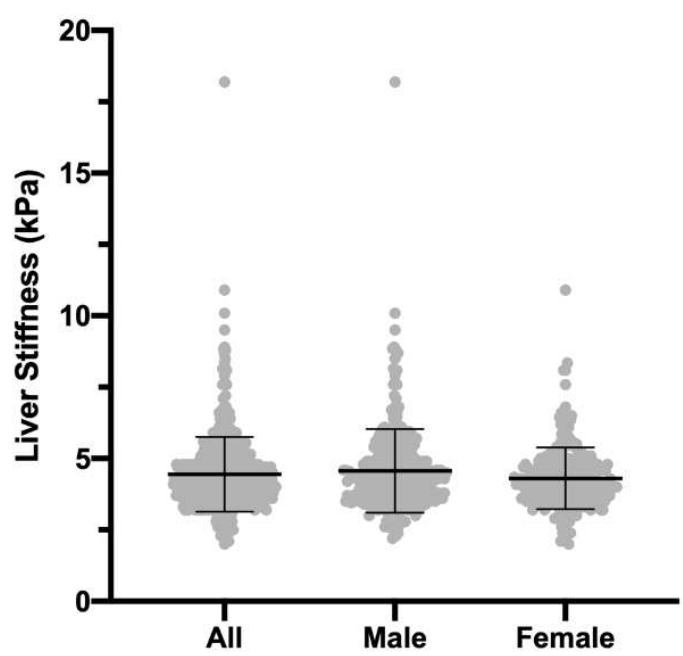

B

\section{LSM (< 3 years old)}

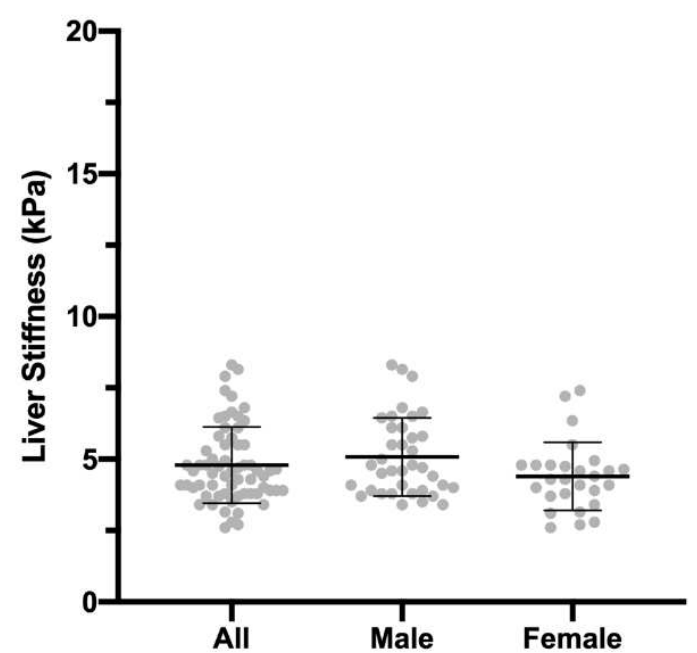

Figure 2. Dot plots of LSM for children for the (A) older ( $\geq 3$ years old) and (B) younger ( $<3$ years old) groups stratified by sex. The solid line represents the mean and the bars represent standard deviation. 


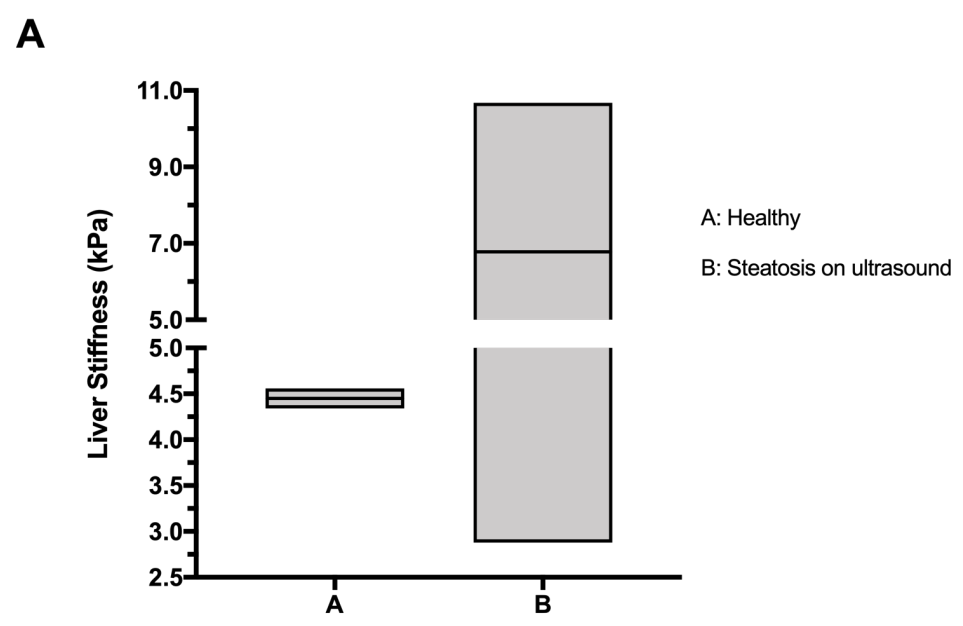

B

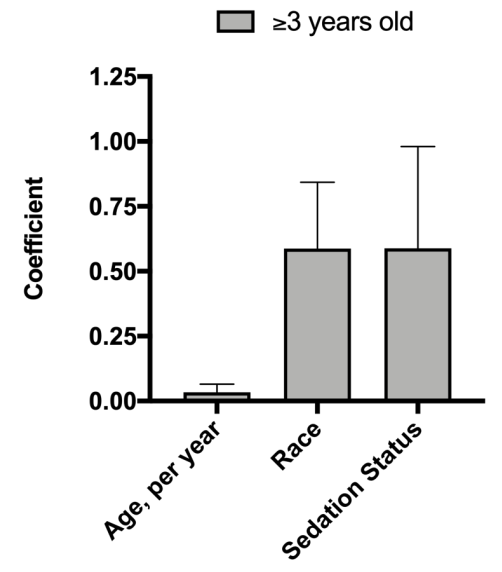

C

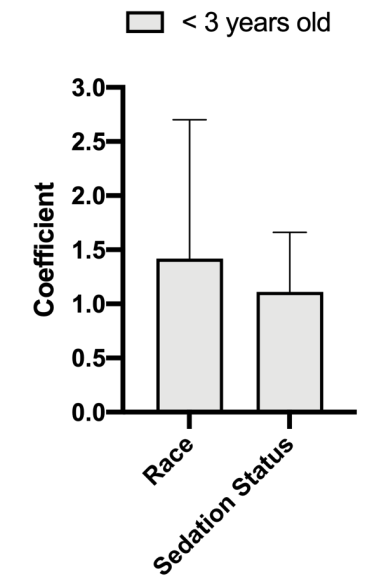

D

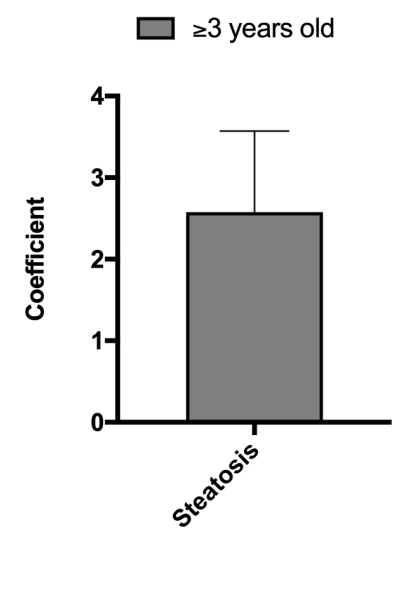

Figure 3. Summary of major results in this IPD meta-analysis. (A) LSM measurements for children $\geq 3$ years old who were truly healthy and who had steatosis on ultrasound. Boxes represent $95 \% \mathrm{Cl}$ and lines represent the mean. (B) Significant factors influencing LSM in children < 3 years old; (C) Significant factors influencing LSM in children $\geq 3$ years old; (D) Significant factors influencing LSM in children $\geq 3$ years old with information regarding presence or absence of steatosis on ultrasound. Bars represent means and error bars represent $95 \% \mathrm{Cl}$. 